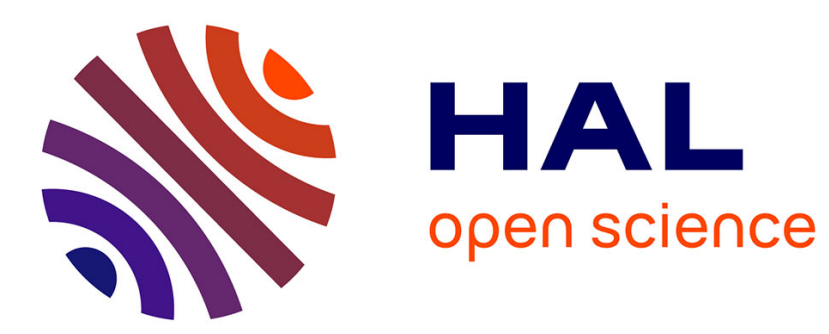

\title{
Mining Spatial Gradual Patterns: Application to Measurement of Potentially Avoidable Hospitalizations
}

Huu Tu Ngo, Véra Georgescu, Anne Laurent, Thérèse Libourel Rouge, Grégoire Mercier

\section{- To cite this version:}

Huu Tu Ngo, Véra Georgescu, Anne Laurent, Thérèse Libourel Rouge, Grégoire Mercier. Mining Spatial Gradual Patterns: Application to Measurement of Potentially Avoidable Hospitalizations. SOFSEM, Jan 2018, Krems, Austria. pp.596-608, 10.1007/978-3-319-73117-9_42 . hal-01828664

\section{HAL Id: hal-01828664 \\ https://hal.science/hal-01828664}

Submitted on 9 Oct 2019

HAL is a multi-disciplinary open access archive for the deposit and dissemination of scientific research documents, whether they are published or not. The documents may come from teaching and research institutions in France or abroad, or from public or private research centers.
L'archive ouverte pluridisciplinaire HAL, est destinée au dépôt et à la diffusion de documents scientifiques de niveau recherche, publiés ou non, émanant des établissements d'enseignement et de recherche français ou étrangers, des laboratoires publics ou privés. 


\title{
Mining Spatial Gradual Patterns: Application to Measurement of Potentially Avoidable Hospitalizations
}

\author{
Tu Ngo ${ }^{1,2}$, Vera Georgescu ${ }^{2}$, Anne Laurent $^{3}$, Thérèse Libourel ${ }^{4}$, and \\ Grégoire Mercier $^{2}$ \\ 1 Dept. of Information and Communication Technology, Univ. of Science and \\ Technology of Hanoi, Vietnam \\ ${ }^{2}$ Economic Evaluation Unit, University Hospital of Montpellier, France \\ 3 LIRMM, University of Montpellier, France \\ 4 Espace-Dev, University of Montpellier, France
}

\begin{abstract}
Gradual patterns aim at automatically extracting covariations between variables of data sets in the form of "the more/the less" such as "the more experience, the higher salary". This data mining method has been applied more and more in finding knowledge recently. However, gradual patterns are still not applicable on spatial data while such information have strong presence in many application domains. For instance, in our work we consider the issue of potentially avoidable hospitalizations. Their determinants have been studied to improve the quality, efficiency, and equity of health care delivery. Although the statistical methods such as regression method can find the associations between the increased potentially avoidable hospitalizations with its determinants such as lower density of ambulatory care nurses, there is still a challenge to identify how the geographical areas follow or not the tendencies. Therefore, in this paper, we propose to extend gradual patterns to the management of spatial data. Our work is twofold. First we propose a methodology for extracting gradual patterns at several hierarchical levels. In addition, we introduce a methodology for visualizing this knowledge. For this purpose, we rely on spatial maps for allowing decision makers to easily notice how the areas follow or not the gradual patterns. Our work is applied to the measure of the potentially avoidable hospitalizations to prove its interest.

Keywords. Data Mining, Gradual Patterns, Spatial Maps, Cartography Visualization, Potentially Avoidable Hospitalizations.
\end{abstract}

\section{Introduction}

\subsection{Problem Statement}

It is often estimated that over $90 \%$ of the information integrate spatial information. In many cases, this important component has not yet been taken into account for specific designs and implementations. However, such spatial information are currently taking more and more importance with the emergence of 
Internet of Things and popular applications integrating spatial information (e.g., Google maps).

In this context, it is important to analyze the information at several levels of granularity with upscaling and downscaling features. Public policies are especially targeted in such analyses as actions can be taken at different levels of administrative entities (cities, regions, states,...). In our context, we focus on potentially avoidable hospitalizations. We aim at extracting gradual patterns so as to analyze the co-variations of descriptors and highlight some actionable features. Gradual patterns are of the form the more/less $A_{1}, \ldots$, the more/less $A_{n}$. Such co-variations can not easily be aggregated through spatial levels of granularity and some knowledge that could be relevant at some level could be called into question at some other level. Thus, we aim at helping decision makers to navigate through the most relevant features and levels of granularity.

In this paper, we introduce a generic method for extracting and analyzing gradual patterns from spatial data at several levels of granularity. Our method is based on the extraction of gradual patterns. One key point is then to deal with aggregation from one level to the upper level.

This method is applied to a real case for analyzing potentially avoidable hospitalization that are both societal and financial issues in public policies.

\section{$1.2 \quad$ Use Case}

The use case we are working on is meant at helping decision makers from the public health system. Typically, in France, the public health decision makers can have an impact on the determinants related to health care such as the density of physicians, nurses, or the density of hospital beds, but they have no influence on socio-economic determinants such as poverty and education. Potentially avoidable hospitalizations (PAH) are hospital episodes that could have been avoided if patients had received timely and effective primary care. Avoiding these admissions by improving access to primary care could result in a substantial decrease in costs and could enhance patients' quality of life [1]. The main objective for avoiding these hospitalizations is to enhance the health care services where and when it is feasible by working on actionable features.

In this work, we consider two levels of granularity: geographic code and department levels where the patients live. This geographic code level corresponds roughly to the postal code level $(\mathrm{n}=5,590)$ while the department level is similar to the county level in the United States $(\mathrm{n}=96)$. It should be noted that French overseas territories were excluded because most data is lacking.

In addition, this research is conducted on the datasets with the age- and sexstandardized rates of PAH. The standardization for the rates of PAH is necessary to allow for an unbiased comparison between spatial elements [5]. The datasets are provided by the Ministry of Health data that is the national hospital discharge database (fr. Programme de Médicalisation des Systèmes d'Information PMSI). It includes data from all French hospitals, whether public or private, and for all payers. Discharge data are obtained at the patients' residence geographic 
code level. The reliability and validity of the PMSI are high for various acute and chronic conditions, especially since 2007 [6]. This research has been approved by the National Committee on Information Technology and Civil Liberties (Reference number CNIL/DE-2014-134)

The PAH potential determinants including the rates of ambulatory care nurses (per 1,000 people), the rates of general practitioners, the rates of specialist physicians, the annual median patient incomes, percentage of education level beyond high school, the mortality ratios, and the rates of acute care hospital beds had been collected from French Ministry of Health and from the National Institute for Statistics and Economic Studies (INSEE).

This research is the next step of one of the previous finding [1] of a French national project on PAH funded by the French Ministry of Health. That previous research has found the associations between the standardized rates of PAH with higher mortality ratio, lower density of ambulatory care nurses, lower median income, and lower education levels and other effects [1].

Those findings are useful for policy makers. However, that research does not help answer which areas to increase the rate of the nurses, for instance, in order to reduce the numbers of PAH. Hence, another research should be conducted for the answers and it is the main aim of this research.

\subsection{Approach Proposal}

The approach proposed in this paper is an extension of [4]. It considers the extraction of gradual patterns at several spatial granularity levels that are then compared and plotted on spatial maps. The rest of this paper details this approach. We first detail the methodology proposed, described here as a workflow integrating the use of a spatial database and spatial maps. We also discuss how to deal with the extraction and study of gradual patterns at several granularity levels.

The approach we introduce here is generic. Although it has been tested and assessed on real data from French public health, it can be used on any data containing spatial information and which attribute domains are partially ordered so as to allow gradual patterns to be extracted, as recalled below with the basic concepts associated to gradual patterns.

\section{Related Works}

\subsection{Around Gradual Patterns}

Gradual patterns consider tendencies in terms of correlation of the attribute variations [2]. A gradual pattern is in the form of "the more/the less $A_{1}, \ldots$, the more/the less $A_{n}$ " such as "the more experience, the higher salary" or "the older a subject, the less his memory".

A gradual item is defined as a pair $(\mathrm{i}, \mathrm{v})$ in which $\mathrm{i}$ is an attribute of the given dataset and $\mathrm{v}$ is variation with $\mathrm{v} \in\{\uparrow, \downarrow\}$ in which $\uparrow$ stands for increasing 
variation and $\downarrow$ stands for decreasing variation. A gradual pattern is a set of gradual items denoted by $\left\{\left(i_{1}, v_{1}\right), \ldots,\left(i_{n}, v_{n}\right)\right\}$.

For example, given that we have a dataset with three attributes: A, B, and $\mathrm{C}$ as shown in table $1,(\mathrm{~A}, \downarrow)$ is a gradual item and $\{(\mathrm{A}, \downarrow),(\mathrm{C}, \uparrow)\}$ is a gradual pattern.

Table 1. An example dataset

\begin{tabular}{llll}
\hline Objects & A & B & C \\
\hline Obj 1 & 4 & 6 & 12 \\
Obj 2 & 1 & 9 & 13 \\
Obj 3 & 2 & 10 & 10 \\
Obj 4 & 5 & 8 & 11 \\
Obj 5 & 3 & 7 & 14 \\
\hline
\end{tabular}

Support of Gradual Patterns. The support value of a gradual pattern indicates to which extent it is true in a given dataset. There are several ways to compute such a value $[2,3]$. In this paper, we consider the approach based on precedence graphs.

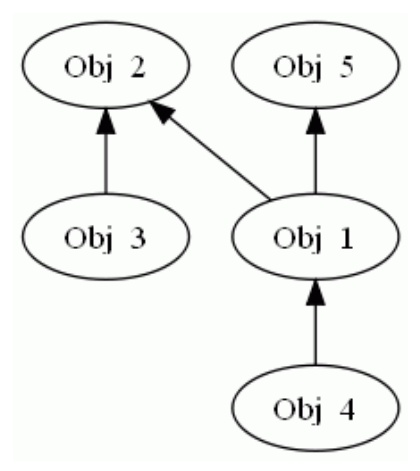

Fig. 1. Precedence graph for the gradual pattern $\{(\mathrm{A}, \downarrow),(\mathrm{C}, \uparrow)\}$ with the example dataset.

A precedence graph is considered for one given gradual pattern and represents the partial order of the objects from the database through paths. For instance, when considering the data from Table 1 and the gradual pattern $\{(\mathrm{A}, \downarrow),(\mathrm{C}, \uparrow)\}$, then Obj $3 \prec \operatorname{Obj} 2$ as $2>1$ and $10<13$.

The support value of a gradual pattern is given by the maximum length of all paths from the precedence graph over the total number of objects. 
Returning back to the example precedence graph (Fig. 1), the value of maxLength is 3 and there are 5 objects. Hence, we have:

$$
\operatorname{Support}(\{(\mathrm{A}, \downarrow),(\mathrm{C}, \uparrow)\})=\frac{3}{5}=0.6
$$

A gradual pattern is said to be frequent if its support is higher than a given support threshold.

Gradual Patterns and Spatial Information. Gradual patterns have been applied in various application domains, as biology, psychology, etc. They have also been studied in the framework of spatial information [4] which proposes a method to visualize on spatial maps the information about to which extent a geographical object (e.g., a city) participates in each gradual pattern. On the other hand, each gradual pattern can be represented by a precedence graph that indicates which paths a spatial object belongs to. For example, on the precedence graph (Fig. 1), [Obj 2] are on two paths: The first one is [Obj 4] $\rightarrow[\mathrm{Obj} 1] \rightarrow$ $[\mathrm{Obj} 2]$ and the other one is $[\mathrm{Obj} 3] \rightarrow[\mathrm{Obj} 2]$. The lengths of these paths are 3 and 2 respectively.

Definition. The item support value of an object is the max length of all the paths the object is on.

Back to the example above, the item support value of [Obj 2] is 3 because the lengths of all the paths [Obj 2] is on are $\{3,2\}$ as mentioned above.

On the other side, since our approach is applied on several hierarchical levels of the spatial objects, there are insider-level objects and container-level objects (in our case, they are geographic codes and department codes respectively).

Definition. The follow-gradual pattern (or Follow-GP) insiders of a container-level object (denoted container) are the insider-level objects (denoted insiders) in the container having GP item support values that are not smaller than a user-defined threshold.

Later in this paper, we will present a use case of this definition when we discuss about aggregation maps.

Spatial Maps. Spatial maps are powerful to highlight information related to locations [10]. There are several types of spatial maps such as choropleth and symbol maps $[10,13]$. However, in the scope of this paper, introduce choropleth maps. Choropleth maps use colors to represent the data associated with an area by filling the shape of that area with different colors. The data could be categorical or numerical. With categorical data, choropleth maps (categorized maps in QGIS) use a different color for each category. When there are only two categories, these maps can be called binary maps as the two categories could be converted into binary values $(0$ and 1$)$. With numerical data, choropleth maps (graduated maps in QGIS) use color progression for the different ranges of data with the principle that the darker colors the higher values of data. In this study, we use these choropleth maps (with QGIS) for the knowledge visualization. 


\subsection{Multiple-level Analysis of Spatial Information}

Statistics. Statistical methods have been developed and applied widely in data analysis in many domains such as health sciences $[11,12]$. In our previous work [1], a multilevel mixed model (regression model with two hierarchical levels) was used to assess disparities in PAH in France in 2012 and analyze their determinants. The result of the research has indicated that the increased PAH were associated with higher mortality, lower density of acute care beds and ambulatory care nurses, lower median income, and lower education levels. It also suggests that primary care organizations play a role in geographic disparities in PAH [1]. However, this statistical method did not take into account the spatial information.

SOLAP. While On-Line Analytical Processing (OLAP) technologies allow fast, easy, and interactive exploration and analysis of data without any expert assistance $[8,7]$, they are not optimized to spatial data. On the other hand, Geographic Information Systems (GIS) are powerful tools for detailed spatial data analysis, but they are not meant to support analytical needs which mostly require summarized information, aggregated data, trends analysis, spatio-temporal comparisons, interactive exploration of data, geographic knowledge discovery in large amounts of data, etc. [9]. The solution of combining the strengths of GIS and the strengths of OLAP tools gives birth to Spatial OLAP (SOLAP) technologies [8]. Like OLAP, these decision making technologies allow easier and faster navigation of geo-spatial databases relying on several levels of information granularity, cross-tabulated data, explicit spacetime integration and more tightly integrated modes of visualization [7]. As SOLAP technologies are very promising, they have been applied in many domains such as surveillance of climate-related health vulnerabilities [9] or public debate [7]. Related to our work, SOLAP technologies could be an interesting extension to our project.

\section{Extracting Spatial Gradual Patterns}

As mentioned in the Introduction section, our goal is to extract associations between potential determinants and spatial objects and to visualize the results with spatial maps to help decision makers to navigate through hierarchical levels.

Considering our use case, we aim in particular at retrieving the potential determinants impacting the rates of $\mathrm{PAH}$ by extracting gradual patterns from different levels of spatial information (geographic codes and department codes). The patterns are then plotted on spatial maps as a reference for the decision makers. In this paper, we present our approach to achieve that goal.

\subsection{Process Flow}

Our implementation approach includes several steps that are described in the process flow in the figure ?? and detailed below. 
Step 1: Find gradual patterns at several granularity levels. At this first step of the process the gradual patterns are extracted at all granularity levels (geographic code and department levels in our use case). Given a support threshold, the output of this step is a list of frequent gradual patterns for every level with their support values.

Step 2: Select gradual patterns for mining. At this step, experts help select some gradual patterns from the output of the first step. A gradual pattern is selected based on both its relevance and the inclusion of actionable variables and its high gradual pattern support value.

Step 3: For every selected gradual pattern, find item support values of spatial objects. At this third step, for every selected gradual pattern, the item support value is computed of every element from every granularity level (e.g., every geographic code and every department code).

Step 4: Find number of follow-GP insiders for every containerlevel element. This step is only processed at the insider-level. In this step, for every selected gradual pattern, a threshold is defined for the item support values. In addition, a join table contains an insider (e.g., geographic code) is in which container (e.g., department). The output of this step is a dataset with the number of follow-GP insiders for every container. This output is used to update the shape file ${ }^{1}$ at the container-level.

Step 5: Update findings into the shape files at all levels. At this step, all the findings found on the previous step for all granularity levels will be updated into the corresponding shape files (geographic codes and departments for our use case). The findings include the item support values of the spatial objects and number of follow-GP insiders for container-level objects.

Step 6: Visualize the findings with the shape files by GIS software. In this step, any GIS software such as QGIS can be used to visualize the findings on spatial maps with the shape files.

\section{$3.2 \quad$ Use Case}

As mentioned in the Introduction section, we have applied this approach to our real datasets that have two hierarchical levels: geographic codes $(n=5,590)$ and department codes $(\mathrm{n}=96)$. In this section, we present the obtained results. For this purpose, an $\mathrm{R}$ package has been developed.

At the geographic code level, the following PAH potential determinants are available:

- Rates of general practitioners per 1,000 people (denoted as Generalists)

- Annual median incomes (denoted as Median income)

- Percentage of education level beyond high school (denoted as Education)

- Rates of ambulatory care nurses per 1,000 people (denoted as Nurses)

\footnotetext{
${ }^{1}$ Popular geo-spatial vector data format for geographic information system (GIS) software
} 
- The rates of ambulatory care specialists per 1,000 people is also available, but there are many geographic codes in which there are no specialists, hence we did not include it when we search for the gradual patterns.

At department code level, besides the potential determinants we have at geographic code level, we also have the data of the followings:

- Standard mortality ratio (denoted as Mortality ratio)

- Number of acute care hospital beds per 1,000 people (denoted as Beds)

- Percentage of CMU-c recipients ${ }^{2}$ (denoted as CMUc recipients)

Select Gradual Patterns for Mining. The Find Gradual Patterns step has generated the list of gradual patterns with their support values. From that list, the experts have selected the following gradual patterns for mining based on their high support values and their relevance to the purpose of the project.

At the geographic code level:

- $\{$ (Median income, $\downarrow$ ), (PAH, $\uparrow)\}$ (denoted as INC in Fig. 7 and Fig. 8)

- $\{$ (Education, $\downarrow$ ), (PAH, $\uparrow)\}$ (denoted as EDU in Fig. 7 and Fig. 8)

- $\{($ Nurses,$\downarrow),(\mathrm{PAH}, \uparrow)\}$ (denoted as NUR in Fig. 7 and Fig. 8)

At the department level:

- $\{($ Nurses,$\downarrow),(\mathrm{PAH}, \uparrow)\}$

- $\{$ (Mortality ratio, $\uparrow),(\mathrm{PAH}, \uparrow)\}$

- $\{($ Generalists,$\downarrow),(\mathrm{PAH}, \uparrow)\}$

- $\{$ (Specialists,$\downarrow),(\mathrm{PAH}, \uparrow)\}$

- $\{($ Education,$\downarrow),(\mathrm{PAH}, \uparrow)\}$

Find Item Support Values. In this step, at each hierarchical level, for every selected gradual pattern, we need to find the item support value of every spatial element. For the purpose of demonstration, in this paper, we only present the item support values of the gradual pattern $\{($ Nurses,$\downarrow),(\mathrm{PAH}, \uparrow)\}$. For the purpose of visualization, we divided these values into 5 groups for each level, at both the geographic code (Fig. 2) and department code (Fig. 3) levels.

\section{Dealing with Spatial Maps and Hierarchies}

\subsection{Navigating through Hierarchical Levels}

In this section, we present how hierarchies can be dealt with in the context of spatial data and gradual patterns. In our approach, we take advantages of the current GIS software (QGIS in our case) to navigate through hierarchical levels. In particular, we use choropleth maps to plot the gradual patterns at each level as layers (as in QGIS). The navigation bar of the GIS software allows us to easily switch among the maps (rolling up or down between the hierarchical

\footnotetext{
${ }^{2}$ In France, CMU-c recipients are people who are given special rights for a free complementary health care complementary insurance.
} 


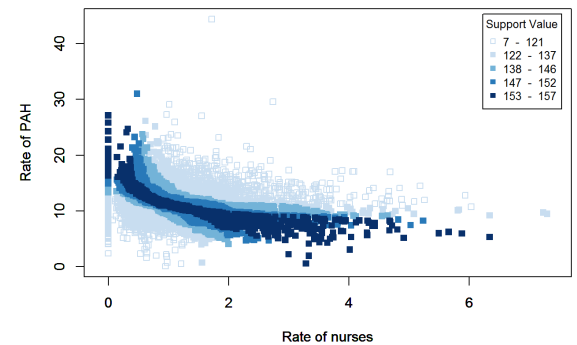

Fig. 2. Item support values of $\{$ (Nurses, $\downarrow)$, $(\mathrm{PAH}, \uparrow)\}$ at geographic code level

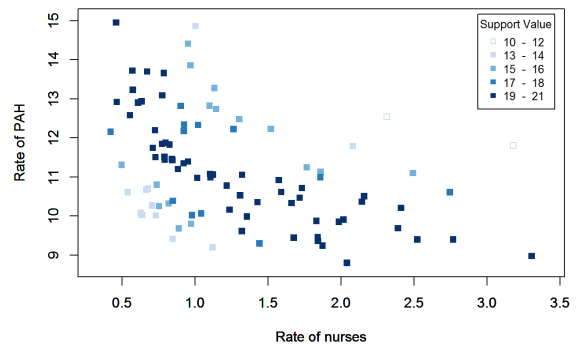

Fig. 3. Item support values of $\{($ Nurses,$\downarrow)$, $(\mathrm{PAH}, \uparrow)\}$ at department code level

levels). In addition, We can pre-compute the aggregation values for the higher level (department in our case) from the lower level one (geographic codes in our case). The aggregation values are computed based on the number of followGP insiders (step 4 in the process flow, Fig. ??). The aggregation values show how insider-level elements of a container-level object participate in a selected gradual pattern. Because the gradual patterns are computed independently at each spatial level, the aggregation values work like the connecting values between the two hierarchical levels. Therefore, by visualizing these values, we would find the valuable information in analysis of hierarchical spatial data.

\subsection{Use Case}

In our work, we implemented two aggregation values. The first one is to show percentage of geographic codes inside a department following a selected gradual pattern. The second one is to display the best gradual patterns in each department. The best gradual pattern is simply defined is the gradual pattern whose number of follow-GP is the biggest compared with the other gradual patterns.

As we have mentioned, for every selected gradual pattern, we need to find the number of follow-GP geographic codes for every department. In order to do that, thresholds are needed. In our case, the experts have selected the following thresholds (Table 2).

Table 2. Thresholds for the numbers of follow-GP insiders

\begin{tabular}{llc}
\hline$\#$ & Gradual pattern & Threshold \\
\hline 1 & $\{($ Median income,$\downarrow),(\mathrm{PAH}, \uparrow)\}$ & 165 \\
2 & $\{($ Education,$\downarrow),(\mathrm{PAH}, \uparrow)\}$ & 161 \\
3 & $\{($ Nurses,$\downarrow),(\mathrm{PAH}, \uparrow)\}$ & 153 \\
\hline
\end{tabular}

After setting the threshold for each gradual pattern, the number of followGP geographic codes of every department can be obtained as shown in the same template (Table 3). 
Table 3. Number of follow-GP geographic codes of department with gradual pattern $\{($ Nurses,$\downarrow),(\mathrm{PAH}, \uparrow)\}$

\begin{tabular}{lll}
\hline$\#$ & Department code & Number of follow-GP \\
\hline 1 & 01 & 6 \\
2 & 13 & 25 \\
3 & 02 & 10 \\
$\ldots$ & $\ldots$ & $\ldots$ \\
\hline
\end{tabular}

\subsection{Finding Visualization on Spatial Maps}

As mentioned in the process flow (Fig. ??), in order to visualize the findings above, we need to import them into the corresponding shape files at the hierarchical levels. This step can be conducted by SQL commands on database management system (DBMS) with spatial extension (postgresql and postgis in our case).

Graduated Maps for Item Support Values. These maps (Fig. 4 and Fig. 5) facilitate the decision makers to find out how an area (geographic code and department) follows a selected gradual pattern through its item support value, the higher item support value the better it follows the gradual pattern.

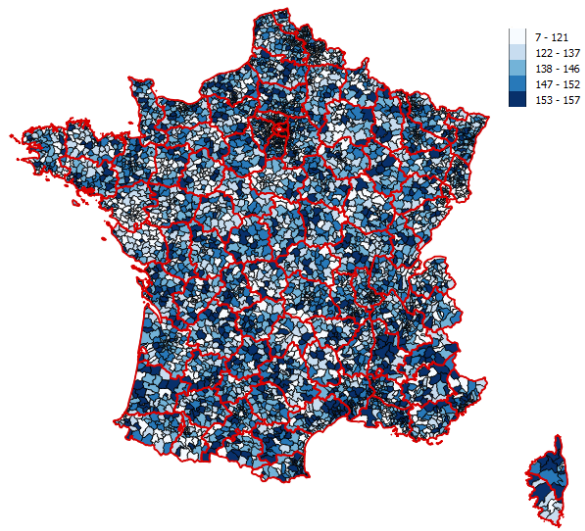

Fig. 4. Graduated map for item support values of $\{($ Nurses,$\downarrow),(\mathrm{PAH}, \uparrow)\}$ at geographic code level

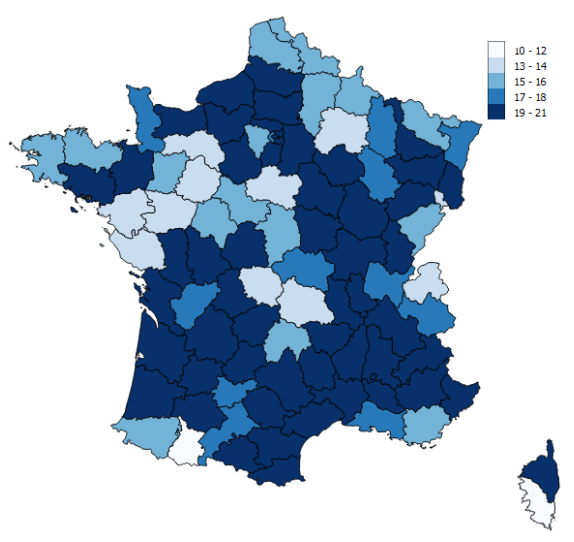

Fig. 5. Graduated map for item support values of $\{($ Nurses,$\downarrow),(\mathrm{PAH}, \uparrow)\}$ at department code level

Aggregation Maps at Container-Level from the Insider-Level. In these maps, we visualize the aggregation values on spatial maps. In our case, we implemented two types of the aggregation maps: 


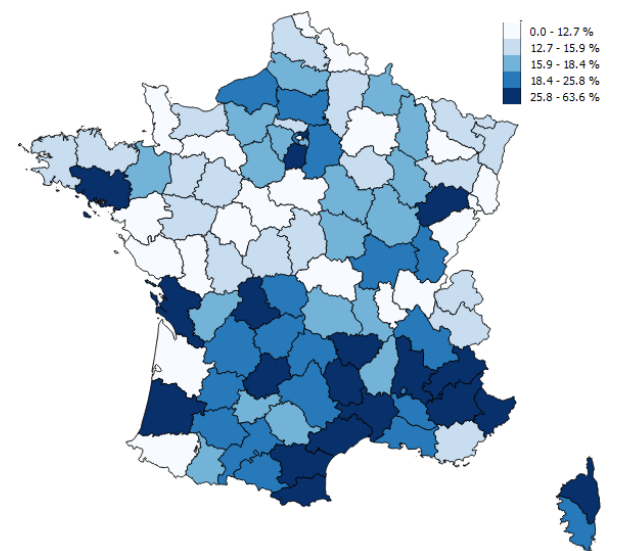

Fig. 6. Percentage of geographic codes inside department codes following $\{($ Nurses,$\downarrow),(\mathrm{PAH}, \uparrow)\}$

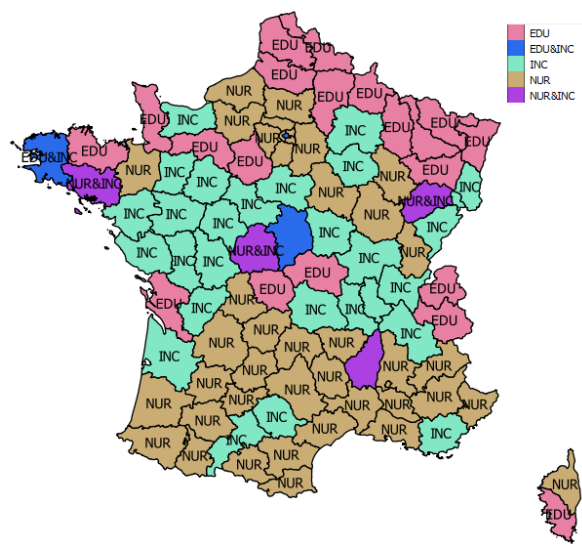

Fig. 7. Best gradual patterns at each department code

The first type is to display the percentage of geographic codes inside department codes following a selected gradual patterns (Fig. 6).

The second aggregation map is the best gradual pattern map (Fig. 7). For example, on the map above (Fig. 7), EDU\&INC indicates that the departments have the have the same numbers of follow-GP geographic codes for $\{$ (Education, $\downarrow$ ), $(\mathrm{PAH}, \uparrow)\}$ and $\{($ Median income,$\downarrow),(\mathrm{PAH}, \uparrow)\}$

Multiple-Gradual-Pattern Maps. This type of spatial maps (Fig. 8) is to display multiple gradual patterns on a same map. This type of maps could be useful for the decision makers as they can analyze the association between many different determinants on the spatial objects. In particular, in our case, the decision makers want to see which gradual patterns the geographic codes follow.

\section{Conclusions}

In this paper, we have presented an approach of using gradual pattern method to not only find the correlation of the attribute variations as the regression method does but also to mine geographical data and to identify how an object participates in the associations between attribute variations through the gradual pattern item support values of spatial elements. The visualization of the spatial areas is crucial.

In our work, we have applied our method to study which areas participate in the association between primary care and PAH. This work is of high interest to national- and regional-level health authorities. Indeed, the latter might focus their efforts and investments on these areas in order to maximize the efficiency at the health system level. 


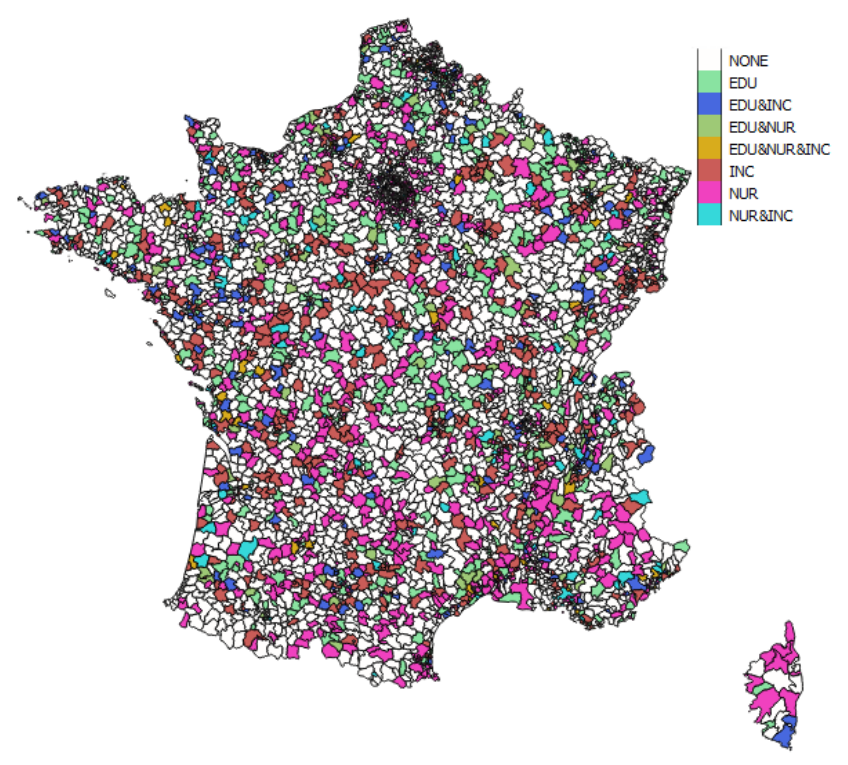

Fig. 8. Multiple-gradual-pattern map at geographic code level

This paper describes the method of using spatial maps with shape files and GIS software as tools for the decision making people. In particular, this paper gives some ideas of visualizing the gradual pattern support values of the geographic codes and the departments the on the spatial maps. These maps might be the useful references for decision makers at the French Ministry of Health when they want to reduce the numbers of PAH in France.

Further work includes the integration of these proposals in the context of Spatial OLAP systems in order to provide end-users with integrated fluid solutions for navigating through the spatial data and hierarchies with such complex measures (gradual patterns).

\section{Acknowledgements}

We would like to thank University of Science and Technology of Hanoi (USTH) and the DIM department from the CHU of Montpellier for funding this work.

\section{References}

1. Mercier, G., Georgescu V., Bousquet J.: Geographic variation in potentially avoidable hospitalizations in France. Health affairs. 34, 836-843 (2015)

2. Laurent, A., Lesot, M.-J., Rifqi, M.: GRAANK: Exploiting rank correlations for extracting gradual itemsets. In: FQAS '09 Proceedings of the 8th international conference on flexible query answering systems. Roskilde, Denmark (2009) 
3. Di-Jorio, L., Laurent, A.,Teisseire, M.: Mining frequent gradual itemsets from large databases. In: IDA '09 Proceedings of the 8th international symposium on intelligent data analysis: Advances in intelligent data analysis VIII. Lyon, France (2009)

4. Aryadinata, Y., Lin, Y., Barcellos, C., Laurent, A., Libourel, T.: Mining epidemiological dengue fever data from Brazil: a gradual pattern based geographical information system. In: Proceeding of 15 th international conference on information processing and management of uncertainty in knowledge-based systems, IPMU 2014, Part II. Montpellier, France (2014)

5. Nelson, G. S.: Reporting healthcare data: Understanding rates and adjustments. In: PharmaSUG 2014 conference proceedings. San Diego, United States (2014)

6. Goldberg, M., Coeuret-Pellicer, M., Ribet, C., Zins, M.: Epidemiological studies based on medical and administrative databases : a potential strength in France. Medicine sciences. 28(4), 430-4 (2012)

7. McHugh, R., Roche, S., Bedard, Y.: Towards a SOLAP-based public participation GIS. Journal of environmental management. 90(6), 2041-2054 (2008)

8. Rivest, S., Bdard, Y., Proulx, M.-J., Nadeau, M., Hubert, F., Pastor, J.: SOLAP technology: Merging business intelligence with geospatial technology for interactive spatio-temporal exploration and analysis of data. Journal of the international society for photogrammetry and remote sensing (ISPRS). 60(1), 17-33 (2005)

9. Bernier, E., Gosselin, P., Badard, T., Bdard, Y.: Easier surveillance of climaterelated health vulnerabilities through a Web-based spatial OLAP application. International journal of health geographics. 8(18) (2009)

10. Magnuson, L.: Data visualization: A guide to visual storytelling for libraries. Rowman and Littlefield publishers, Maryland, United States (2016)

11. Auget, J.-L., Balakrishnan, N., Mesbah, M., Molenberghs, G.: Advances in statistical methods for the health sciences: Applications to cancer and AIDS studies, genome sequence analysis, and survival analysis (statistics for industry and technology). Birkhuser,Basel, Switzerland (2007)

12. Ott, L. R., Longnecker, M.: An introduction to statistical methods and data analysis. Duxbury, Australia (2001)

13. Zhang, L., Guo, Q., Jiao, L.: Design and implementation of decision-making support system for thematic map cartography. The international archives of the photogrammetry, remote sensing and spatial information science. 37. (2008) 